To the Editors:

\title{
Estimation of provider cost and the out of pocket expenditure for cataract surgery at a base hospital
}

\author{
C Perera', D De Silva², L R P Perera ${ }^{1}$
}

Ceylon Medical Journal 2016; 61: 200

http://doi.org/10.4038/cmj.v61i4.8395

Cataract surgery is one of the commonest surgical procedures carried out in the elderly and it improves quality of life of the affected. Currently it is relatively risk free, speedy and leads to dramatic recovery during the post-operative period.

A descriptive cross sectional study was conducted after obtaining approval from Ethics Review Committee, Faculty of Medicine, Ragama. Permission was obtained from the consultant ophthalmologist of the unit. A pretested interviewer administered questionnaire was used to collect data. The study was carried out from April 2015 to June 2015. All patients $(n=202)$ who were admitted for surgery during the study period were included in the study.

The cost of management of cataract includes all costs from time of admission up to discharge after cataract surgery. The cost included cost of inward treatment, investigations (FBS, ECG) and total operation room cost. Salaries and the consumable prices were estimated according to cost in April 2015. The out of pocket expenditure which included direct and approximate indirect household costs were included in the estimation of cost. Direct costs included cost of travel, drugs and investigations borne by the patient. Indirect cost included cost of unemploy-ment due to illness or seeking and receiving treatment.

The mean age of the patients was 65 years. Cataract surgical acceptance was high in females. Sample consisted of $62 \%$ females and $38 \%$ males. Average daily income of the study population was SLR. 384 (Annual income US\$1040).

Total provider cost for cataract surgery was SLR15668. Two and a half days of hospital stay accounted for SLR 8515 (54\%) of the provider cost. Operating room cost accounted for $44 \%$ of the total cost. Total cost of staff services for a cataract surgery was SLR 6365 (41\%) of the provider cost. The cost of the phaco machine and the related equipment accounted for $22 \%$ of the provider cost. Human resources and technologically advanced instrument cost accounted for $63 \%$ of the institutional cost. Therefore efficient management of human resources and technological advancement was a priority for health care managers to reduce the provider cost of cataract surgery.

Total out of pocket expenditure of a patient undergoing cataract surgery was SLR 25407. Cost of transport, food, investigations, drugs, lens and other consumables and cost of a bystander, accounted for $97 \%$ of out of pocket expenditure. Loss of income accounted for $1 \%$. The total cost of surgery was SLR 41075 (US\$ 310). There is no data from Sri Lanka but a study done in UK in 1999 showed average cost per cataract procedure was $£ 359.89$ (US\$ 415) [1]. The difference in cost may be due to inflation and differences of exchange rates since 1999, and the inequality of the two health systems such as availability of insurance schemes.

The major cost of the cataract surgery is borne by the patient $(62 \%)$. The total cost of cataract surgery is about nine times higher than the total per capita health expenditure (SLR 4392) of Sri Lanka in 2012 [2]. The cost of cataract surgery was about four times the average monthly per-capita income in Sri Lanka (SLR 11 932) [3]. Therefore policy makers should analyse healthcare costing in depth to utilise the resources efficiently and cost-effectively while maintaining quality of service.

\section{Conflicts of interest}

There are no conflicts of interest.

\section{References}

1. Desai P, Reidy A, Minassian DC. Profile of patients presenting for cataract surgery in the UK: national data collection. BrJ Ophthalmol 1999; 83: 893-6.

2. Ministry of Health, Annual Health Bulletin. Ministry of Health, Sri Lanka 2012.

3. Department of Census and Statistics, Household Income and Expenditure Survey 2012/2013 Ministry of Finance and Planning Sri Lanka 2013.

${ }^{1}$ Postgraduate Institute of Medicine, University of Colombo and ${ }^{2}$ Ministry of Health, Sri Lanka.

Correspondence: CP, e-mail: <chinthaka159perera@yahoo.co.uk>. Received 4 April 2016 and revised version accepted 9 July 2016.

This is an open-access article distributed under the terms of the Creative Commons Attribution License, which permits unrestricted use, distribution, and reproduction in any medium, provided the original author and source are credited. 